\title{
Scientific Organizing Committee
}

\author{
Alan H. Batten (Chairman, Canada) \\ Olga Dluzhnevskaya (Russia) \\ Julieta Fierro (México) \\ John B. Hearnshaw (New Zealand) \\ Rajesh K. Kochhar (India) \\ Yoshihide Kozai (Japan) \\ Li QiBin (China) \\ Peter Martinez (South Africa) \\ Derek McNally (United Kingdom) \\ Lesley I. Onuora (United Kingdom/Nigeria) \\ Maria C. Pineda de Carias (Honduras) \\ Morton S. Roberts (United States of America) \\ Major Contributors
}

Toufik Abdelatif, Observatoire d'Alger-CRAAG, BP 63, Bouzaréah 1630, Alger, Algeria.

Helmut A. Abt, Kitt Peak National Observatory, Box 26732, Tucson, Arizona 85726-6732, U.S.A.

M.N. Anandaram, Department of Physics, Bangalore University, Bangalore 560056 , India.

Pulat B. Babadzhanov, Institute of Astrophysics, Dushanbe 734042, Tajikistan. Alan H. Batten, Dominion Astrophysical Observatory, 5071, W. Saanich Rd, Victoria, B.C., Canada, V9E 2E7.

S.P. Bhatnagar, Department of Physics, Bhavnagar University, Bhavnagar, India.

Cynthia P. Celebre, PAGASA (Weather Bureau), 1424 ATB, Quezon Ave, 1104 Quezon City, Philippines.

Khalil Chamcham, King Hassan II University-Ain Chock, Faculty of Science, B.P. 5366, Maarif, Casablanca, Morocco.

Christopher J. Corbally, Vatican Observatory, University of Arizona, Tucson, Arizona 85721, U.S.A.

David L. Crawford, GNAT, Inc., Tucson, Arizona 85716, U.S.A.

Philippe Eenens, Department of Astronomy, University of Guanajuato, Apartado Postal 144, Guanajuato, CP 36000, México.

Cheng Fang, Department of Astronomy, Nanjing University, Nanjing, China. Julieta Fierro, Instituto de Astronomía, UNAM, Apt 70 264, México DF 04510, México.

John B. Hearnshaw, Department of Physics and Astronomy, University of Canterbury, Christchurch, New Zealand.

George Helou, IPAC, Mail Code 100-22, California Institute of Technology, 1200 E. California Blvd, Pasadena, California 91125, U.S.A.

Mary Kay Hemenway, Department of Astronomy, University of Texas, Austin, Texas 78712-1083, U.S.A.

Peter D. Hingley, Royal Astronomical Society, Burlington House, Piccadilly, London W1V ONL, U.K. 
Abendour Irbah, Observatoire d'Alger-CRAAG, B.P. 63, Bouzaréah 1630, Alger, Algeria.

Barrie W. Jones, The Open University, Milton Keynes MK7 6AA, U.K.

B.A. Kagali, Department of Physics, Bangalore University, Bangalore 560 056, India.

Norio Kaifu, National Astronomical University of Japan, Mitaka, Osawa 2-21-1, Tokyo, 181-8588, Japan.

Peter C. Kalebwe, Physics Department, University of Zambia, P.O. Box 32379, Lusaka, Zambia.

Masatoshi Kitamura, National Astronomical Observatory, Mitaka, Tokyo 181-8588, Japan.

D.A. Kovaleva, Institute of Astronomy, 48 Pyatnitskaya St., Moscow 109017, Russia.

Ethleen Lastovica, South African Astronomical Observatory, P.O. Box 9, Observatory, 7935, South Africa.

Koitiro Maeda, Department of Physics, Hyogo College of Medicine

Nishinomiya, Hyogo 663-8501, Japan.

Oleg Yu. Malkov, Institute of Astronomy, 48 Pyatnitskaya St., Moscow 109017, Russia.

Terry J. Mahoney, Scientific Editorial Service, Research Division, Instituto de Astrofísica de Canarias, E-38205 La Laguna, Tenerife, Spain.

Peter Martinez, South African Astronomical Observatory, P.O. Box 9, Observatory 7935, South Africa.

Janet A. Mattei, AAVSO, 25 Birch Street, Cambridge, Massachusets 02138-1205, U.S.A.

Derek McNally, Department of Physical Sciences, University of Hertfordshire, College Lane, Hatfield, Herts., AL10 9AB, U.K.

Geoffrey Munyeme, Department of Physics, University of Zambia, P.O. Box 32379, Lusaka, Zambia.

Jayant V. Narlikar, Inter-University Centre for Astronomy and Astrophysics, Pune 411007, India.

Anas M.I. Osman, National Research Institute of Astronomy and Geophysics, Helwan, Egypt.

Lesley I. Onuora, Astronomy Centre, University of Sussex, Falmer, Brighton BN1 9QJ, U.K.

Jay M. Pasachoff, Hopkins Observatory, Williams College, Williamstown, Massachusets 01267, U.S.A.

John R. Percy, Erindale Campus, University of Toronto, Mississauga, Ontario, Canada L5L 1C6.

Maria C. Pineda de Carias, Central American Observatory of Suyapa, National Autonomus University of Honduras, Tegucigalpa MDC, Honduras.

Sandra Preston, Department of Astronomy, University of Texas, Austin, Texas 78712-1083, U.S.A.

Moedji Raharto, Bosscha Observatory, Lembang 40391, Java, Indonesia.

Kavan U. Ratnatunga, Department of Physics, Carnegie Mellon University, Pittsburgh, Pennsylvania 15213, U.S.A.

Case L. Rijsdijk, South African Astronomical Observatory, P.O. Box 9,

Observatory, 7935, South Africa. 
Nguyen Quang Rieu, Observatoire de Paris, Department DENIRM, 61 Avenue de l'Observatoire, 75014 Paris, France.

Rosa M. Ros, Department of Applied Mathematics IV, Technological University of Catalonia, Jordi Girona 1-3, Modul C3, 08034 Barcelona, Spain. Patricia Rosenzweig, Universidad de Los Andes, Facultad de Ciencias, Departamento de Física, Grupo de Astrofísica Teórica (GAT), Mérida, Venezuela.

Hamid Sadsaoud, Observatoire d'Alger-CRAAG, B.P. 63, Bouzaréah 1630, Alger, Algeria.

Michael S. Snowden, P.O. Box 44, MacPherson Rd, Sinagapore 913402.

Boonraksar Soonthornthum, Sirindhorn Observatory, Department of Physics, Faculty of Science, Chiang Mai University, Chiang Mai 50200, Thailand.

Bernardo M. Soriano Jr, PAGASA (Weather Bureau), 1424 ATB, Quezon Ave, 1104 Quezon City, Philippines.

Yuhua Tang, Department of Astronomy, Nanjing University, Nanjing, China. Noritaka Tokimasa, Nishi-harima Astronomical Observatory Sayocho, Hyogo 679-5313, Japan.

Alexis E. Troche-Boggino, Observatorio Astronomico "Alexis E.

Troche-Boggino", Universidad Nacional de Asunción, Facultad Politécnica, 01 Agencia Postal Campus U.N.A., Central XI, Paraguay.

Alexander V. Tutukov, Institute of Astronomy, 48 Pyatnitskaya St., Moscow 109017, Russia.

Jingxiu Wang, National Astronomical Observatories, Chinese Academy of Sciences, Beijing 100012, China.

Donat G. Wentzel, University of Maryland, College Park, Maryland, 20742-2421, U.S.A. 